Motto: $\quad$ No internal audit without internal control, No internal control without working procedures

No working procedures without risk register

The author

\title{
ESTABLISHING THE DIMENSIONS OF THE INTERNAL AUDIT COMPARTMENT AND THE WAY OF ELABORATING THE RISK REGISTER
}

\author{
Professor PhD Marcel Ghita, marcel.ghita@mfinante.ro, Athenaeum University Bucharest \\ PhD Student Razvan Ghita, Valahia University Târgoviste
}

Abstract: Bearing in mind the fact that through the Law no. 672/2002 regarding public internal audit it is not mentioned the number of internal auditors which will compose the internal audit compartments within the public entities, there are many solicitations for CHUIA to offer explanations regarding this problem.

The dimensions of the audit compartments are established on the basis of risk analysis, a new element, and on the size of the public entity, resulting, thus: directorates, services, bureaus or internal audit compartments.

Because of these considerations, in this material we present the methodology needed to establish the number of internal auditors necessary for assuring the internal audit function within the public entity, starting from the evaluation of its activity, on the basis of the audit plan and, also, on the basis of risk analysis.

A priority in implementing internal control and management system it is represented by the realization of operational procedures for all the activities within the entity. Because of these considerations, CHUFMCS has issued the Order of ministry of public finances no. 1.389/2006 for modifying and completing the Order of ministry of public finances no. 946/2005 through which it's regulated the manner in which working procedures must be elaborated within Romania's public sector.

The article presents the tasks before starting to elaborate the operational procedures and a series of considerations regarding their realization.

In the public sector, internal audit compartment it is organized within public entities, working under general management, according to Law no. 672/2002 regarding public internal audit, and in the private system, internal audit function its organized according to Law no. 3/1990 regarding commercial companies, republished, and internal audit Regulations. ${ }^{1}$

Normative framework doesn't establish the number of auditors within internal audit compartments neither gives a calculation algorithm for their number, but recommends the determination of the internal auditors number on the basis of the risk analysis with which the entity confronts, and in which to bear in mind their evolution, the internal audit compartment's and the submission, for approval, to the general management.

Until general management will assure the determined number of internal auditors on the basis of the risk evaluation analysis, internal auditors will respond for the main risks with which the entity confronts, and the other risks will remain in general management's responsibility.

\footnotetext{
${ }^{1}$ Government Decision for the approval of internal audit regulations no. 88/2007, elaborated by the Chamber of Financial Auditors of Romania, published in the Official Gazette nor. 416/2007.
} 
For establishing the number of internal auditors on the basis of risk analysis, we recommend the evaluation of auditable domain and audit resources, and through their comparison it will be able to establish the required number of internal auditors for assuring the internal audit function within the entity, by passing through the following stages:

a) analysis of the official list of activities, structured by compartments. If such a list doesn't exist or a verification or update is required, we recommend the realization of this list of activities, through repeated demands from employees to have a written list containing job attributions, written on functional compartments within the entity, this list being compared with the functioning and operating code regulation and with job descriptions, for the purpose of completing and updating these charts.

b) dividing each activity in serially primary operations, reasonably detailed, through the description of the process of realizing the activity, from the beginning until is completed, called auditable objects.

c) identifying risks and attaching them to activities' serially primary operations, on the basis of a normative framework analysis, but, also, with the proper experience or practice of those being interviewed;

d) qualitative evaluation of rules and appraise their levels for the purpose of diving them in higher, medium and lower risks, an evaluation recommended to be fulfilled without complicated quantitative formulas;

e) identifying control activities that exist within entities' systems for the delimitation of risks' consequences, by elaborating Internal control questionnaire, but, also, on the basis of field observation;

f) defining greater risks (higher and medium), residual risks, which result from defining risks which have consequences that were not eliminated/diminished by existent control activities within the audited entity through audited activity.

g) defining the multi-annual plan for the public entity, 3-5 times a year, and on the basis of such a plan, it can be devised the internal audit annual plans, structured on missions, which contain:

- auditable fields and activities;

- audited periods;

- the time scale of audit missions in days/human;

- number of required internal auditors.

The number of internal audit missions resulted from this algorithm it will be added the missions recommended by CHIUA or by the Romanian Court of Accounts, required by management, if they have justification, eventually some ad-hoc missions established by the head of the internal audit compartment.

h) defining the internal auditors' necessary resources within the entity, and this number is defined by the centralization of the total number of hours/human allocated on internal audit missions comprised in the internal audit annual plan, on the basis of which it will be established the number of internal auditors needed for the plan's realization.

When we define this calculation we will bear in mind the number of days for vacation, an average number of days for medical leave (5-7 days/year) and the number of continuous professional training (15 working days), which the internal audit compartment head must secure for every internal auditor, according to the Law no. 672/2002 regarding public internal audit.

i) defining the necessary number of internal auditors within the entity resulted as a difference between the internal auditors established within the plan and the internal auditors that work within the internal audit compartment.

Defining such a necessary number of internal auditors will assure the realization of the internal audit function within the internal audit plan for the entity. 
Until the possibility of recruiting internal auditors, a number arise from this calculation, internal audit compartment will supervise major risks, if it's possible and will sustain management in its strategy of recruiting new internal auditors.

According to the internal control Code ${ }^{2}$, comprising internal control management standards within public entities and for the development of managerial control systems, executive management (directors, compartment heads, bureaus heads etc.) has the obligation of identifying risks and to undertake the kind of actions that maintain risks within acceptable limits. For this purpose, it must be maintained a balance between acceptable risk level and the costs of this sort of actions.

Good practice recognized by internal auditors within the field recommends that the general management must have preoccupations for elaborating Risk registers for every compartment, and by centralizing these Register, he will obtain the Risk register for the whole entity.

CHUFMC has elaborated the implementation methodology of internal control standard Risk management ${ }^{3}$, which comprises, as addendum, the Risk register model, mandatory for every public entity from Romania.

Risk register must be realized on the basis of the steps taken when we determined the necessary number of internal auditors within the public entity. A very important element it is represented by the establishment of responsibilities in coordination with Risk Register and, implicative, its regular update.

Risk register comprises residual risks, potential risks, but, also, the risks' history - the risks with which the entity has been confronted in the last period (3-5 years), for every functional compartment.

According to corporate governance principle, concerning transparency, practically every examination/control actions or internal or external audit mission must start with the consultancy of the Risk register of the respective compartment.

\section{Elaborating formal written procedures}

At the same time when the Risk register was elaborated, on the basis of the Order of ministry of public finances no. 946/2005 comprising Internal control Code, the general management will have to pass to the elaboration, lay-down and formalization of working procedures for every activity within the entity. For this purpose, CHUFMC has issued the Order of ministry of public finances no. $1.389 / 2006^{4}$ for the modification and completion of the Order of ministry of public finances no. 946/2005, regarding the elaboration of operational procedures which contain assignations regarding the way to fill the respective documents.

Elaborating working procedures must represent, for every management level within entities, an absolute priority for the employees' responsibility and for the realization, in an unitary fashion, of working tasks, but, also, for the perspective of European funds accessing activities.

Managers, for assuring the beginning of the operational working procedures, which, commonly, are realized at the same time with current working attributions will have to bear in mind the following steps:

a) codifying procedures;

b) prioritizing procedures;

c) establishing the responsible persons for elaborating and updating these procedures.

d) the proper elaboration;

\footnotetext{
2 Methodological Guide for the development of the internal control in the public institutions, www.mfinante.ro, elaborated by CHUFMC within MEF.

${ }^{3}$ www.mfinante.ro, section Preventive financial control/financial control and management system

4 Order for the modification and completion of the Order of ministry of public finances no. 946/2005 approving the Internal Control Code, comprising the management/internal control standards in the public entities and for the development of the managerial control systems
} 
e) formalizing procedures;

f) the corroboration of procedures with job description.

a. For codifying procedures it will be elaborated a logical system scheme, on the basis of the activities' list, and its recommended the usage of the decimal codifying system that allows the subsequent addition of procedures;

b. Prioritizing procedures represents a very important activity for the entity. In general, in Romanian entities, with very few exceptions, we have procedures for $5-10 \%$ from the activities which are realized along the process flux, but in many cases these procedures are not formal or updated.

Because of this, we are aware about the fact that all necessary procedures can't be realized at once, so we can't have procedures for all activities/compartments.

General management together with internal audit compartment must establish a system for prioritizing procedural framework. Thus, we recommend to be given a high priority for realizing current procedures, even daily, then, on a period of time to be realized those that are periodically, then annual.

The prioritizing system of realizing procedures must be approved by the general management for ensuring their elaboration in adequate time.

Nowadays, we can't discuss about European Union integration without the start of a deep process for the realization of procedural framework in all the entities from the Romanian system. Thus, in European practice it is considered a lack of professionalism if there still are entities, activities, programs that function without a procedural system, formalized and approved by every management level.

The international standards for good practice recommend that a member of the board to hold responsibility for creating procedural framework, as quickly as possible (1-2 years).

c. For every procedure there must be a person responsible for elaborating and, subsequent, maintaining, respectively, completing and systematically updating, a function regarding the evolution of procedural activities and legal and normative framework. Good practice recommends that every employee has to detain responsibility for the procedures with which he works. When there are more employees that use the same procedure it is appointed one person to be responsible. This means that the respective person is responsible for the realization and updating of the respective procedure and will be held responsible for implementing and following-up other employees' working procedures.

d. Elaboration of procedures is will be done starting from the legal and normative framework following the total comprise of the specific activities, but also, the inclusion of internal control activities (auto control, mutual control, hierarchic control etc.) following the operational flux, at activities' key-points; establishing responsibilities for every execution level and on phases of the process which is realized (realization, elaboration, approval); respecting the principle of double approval; assuring that what is processed will be transposed into an adequate informative system; establishing ways of archiving documents.

e. procedures, once they are written and formalized meaning that they must contain specific document models, with completed examples and are approved by the general management, must be made mandatory for every category of employees of the entities.

f. Written and formalized procedures must be corroborated with employees' job description, for assuring the complying of these attributes comprised in these procedures with the attributes established by the functioning and operating code regulation of the entity.

We have to mention the fact that the total of written and formalized procedures represents the procedural framework, and this represents a completion of the normative framework and must encompass all the activities, fluxes, processes and operations contained by the legal and normative framework specific for the respective field and must be a sequel of this through practical adaptation to the general normative provisions for the specific entity. 